\title{
UNIVERSITY OF MIAMI NEURO-SPINAL INDEX (UMNI): A QUANTITATIVE METHOD FOR DETERMINING SPINAL CORD FUNCTION
}

\author{
By K. John Klose, Ph.D., ${ }^{1}$ Barth A. Green, M.D., ${ }^{2}$ Robin S. Smith, R.P.T., \\ Rodney H. Adkins, Ph.D. ${ }^{3}$ and ANNE M. MacDonald, R.P.T. \\ ${ }^{1}$ University of Miami School of Medicine; ${ }^{2}$ Acute Spinal Cord Injury, South Florida \\ Regional Spincl Cord Injury System; ${ }^{3}$ South Florida Regional Spinal Cord Injury Project \\ This work is supported in part by the National Institutes of Health, NINCDS \\ \#NSI4468-0I, \#NS-7-2368 and Rehabilitative Services Administration \\ \#I3-P-59258/4-OI.
}

\begin{abstract}
At the University of Miami we have developed a quantitative scale for assessing spinal cord function and have utilised this scale in evaluating acute spinal cord injury patients over the last year. The assessments are performed by specially trained physical therapists who see the patients within an hour of their arrival at the hospital emergency room. The University of Miami Neuro-Spinal Index (UMNI) utilises standard methods of spinal cord functional assessment to produce a numerical value which can be used to determine change in a patient's status, even when the change is minimal. The UMNI yields both a sensory and motor scale which can be dealt with individually or in combination. This scale permits one to compare across patients, quantitative changes in status regardless of the initial level of injury. The UMNI is sensitive to small changes. We are in the process of determining the UMNI's potential as a predictor of outcome.
\end{abstract}

Key words: Sensory testing; Motor testing; Descriptor; Quantitative; Inter-rater Reliability.

\section{Introduction}

HistoRICALly, spinal cord injury was looked upon as a disease which could not be treated. During World War II a different philosophy emerged. At that time Guttmann established the first facility dedicated to the care of spinal cord injured at the Spinal Injuries Centre of Stoke Mandeville General Hospital at Aylesbury, England. Soon after, many European countries followed in establishing centres. Centre care in the United States was initiated by the Veterans Administration during World War II. Initially, the primary emphasis of care was placed on reduction of complications and rehabilitation of the injured patients. More recently interest has focused on the acute care and handling of spinal cord injury patients. Advances in Emergency Medical Services and early care and handling have resulted in the preservation of life for those more severely injured persons formerly expected to die in the acute period following injury. It can also be assumed that many less severe injuries may have a better prognosis due to the absence of secondary injury as a result of improper handling. Many physicians feel that their specific protocols result in a better outcome for patients who have

Correspondence: K. John Klose, Ph.D., Director, CNS Trauma Programs, University of Miami School of Medicine, Department of Neurological Surgery (D4-6), P.O. Box or6960, Miami, Florida 3310I. 
suffered a spinal cord injury. In order to evaluate the efficacy of varying treatment protocols, a sensitive and statistically applicable research instrument is needed. All too often clinical researchers simply state that their specific protocols result in better outcomes without objective quantification.

Spinal cord injured patients have been traditionally classified according to the level of injury and whether the injury was complete or incomplete (Michaelis, I969; Jochheim, I970). This type of description tells something about the status of the patient at any one point in time, but is not an effective research tool. Small changes as a result of a specific intervention or treatment can be masked in a statistical analysis if the change is not great enough to involve a different level of spinal cord function. Cheshire (1970) proposed a classification based on specific functions. This system presents a clearer picture of the patient's clinical status, but has limitations if statistical analyses are desired to compare different methods of care. Other classifications such as the Barthel Index are based on ability to perform activities of daily living (Mahoney et al., 1965; Granger, 1973). The Barthel can be useful in assessing the effectiveness of a rehabilitation programme, but cannot measure neurological changes that may take place from the acute phase through final outcome.

The Yale Spinal Cord Injury Group (Bracken et al., I976) reported an assessment tool which is applicable to acute clinical research. Sensory testing is performed for: superficial pain, light touch, position sense, vibratory sense and deep pain. We are of the opinion that testing of position sense and deep pain is impractical in the early acute stage due to the high incidence of multiple-trauma patients. Additionally testing of position sense for the body trunk areas presents reliability problems. The Yale group tests motor function utilising the five-point standard scoring method. Based on test values, patients are placed in one of seven categories on a sensory severity scale and one of five levels on a motor severity scale. The scale classification levels are used for analytic purposes, where the discharge score is subtracted from the emergency room score. It appears that this method of scaling analysis would not be sensitive to some important functional changes which might take place in some modalities at levels below the highest level of cord involvement. Categorisation of severity on an ordinal rather than a continuous real number scale limits the types of statistical analysis which can be performed on the data.

\section{Method}

Since 1975, researchers at the University of Miami School of Medicine have been working on a numerical index for patient neurological status. The University of Miami Neuro-spinal Index (UMNI) is composed of a sensory and motor scale which are sensitive to small changes. The original instrument has undergone three revisions prior to the version presented here. The specific modalities chosen for testing are based on their anatomical spatial separation within the spinal cord and the ease and reliability of testing.

Degree of spinal cord function is assessed through the use of standard methods, but scored in a unique manner. This method of assessment was chosen for its sensitivity to small changes. The sensitivity of this instrument should prove useful in identifying factors which may help to make subtle differences in patient outcome. Less sensitive descriptors often miss or obscure favourable factors which by themselves produce only small change, but which in combination or sum, make substantial differences. 
The UMNI is composed of two sub-scales, the sensory scale and the motor scale. Scale scores are indicators of overall spinal cord functional capacity within the sensory and motor modalities.

The total score range on the index is $0-460$ where o represents no detectable function and 460 indicates normal function. This total score is the sum of the motor and sensory scales. All UMNI testing is performed by trained physical therapists.

\section{a. Motor function}

The motor scale scoring system is based on muscle testing. There is general agreement in the literature that certain muscle groups are innervated by specific anatomical portions of the spinal cord. The representative muscle groups were chosen on the basis of functional significance as it correlates to level of injury.

Forty-six individual muscle groups are to be tested. Each muscle group is scored on a 0-5 scale (Bickerstaff, I968) where:

$0=$ No function

$\mathrm{I}=$ There is a visible or palpable flicker of contraction, but no resultant movement of limb or joint.

$2=$ There muscle can only make its normal movement when the limb is so positioned that gravity is eliminated.

$3=$ The muscle is able to make its normal movement against gravity, but not against additional resistance.

$4=$ The muscle, though able to make its full normal movement, is overcome by resistance.

$5=$ Normal power.

Since 44 muscle groups are tested on a five-point basis the total motor score ranges from $0-220$.

\section{b. Sensory function}

The sensory scale score is an index of total body sensation. Within the spinal cord two major spatially separated tracts have been identified which carry information from different sensory modalities. The lateral spinothalmic tracts are assessed with pin-prick, while the dorsal columns are assessed with vibration. The rationale of testing both columns is that it provides a more sensitive measure of completeness of injury. Since different skin areas or dermatomes of the body are innervated by specific levels of the spinal cord, this test also determines level of injury for that modality. The dermatome distribution is listed on the UMNI Sensory Evaluation Form.

Sensory testing is performed by the physical therapist using standard procedures (Bickerstaff, 1968). Pain sense is determined by pin-prick and vibration sense by a $256 \mathrm{~Hz}$ tuning fork. Each stimulus is presented bilaterally to each of the dermatome areas innervated by the 30 levels of the spinal cord. Item scores are assigned as follows: $0=$ absent; $I=$ present; but abnormal; $2=$ normal. Sensory indices range from $O=$ no detectable sensation to $240=$ total normal body sensation.

The motor and sensory sub-scale scores can be summed to yield an overall neuro-spinal functional capacity rating. These assessments are made at stated intervals and can be used to chart progression or regression for individuals or groups of patients over a given time period. Additionally, individual assessments are sensitive to small changes, thus the effect of specific interventions can be 


\section{TABLE I}

UMNI Sensory Evaluation Scoring Form, Total score range 0-240

UNIVERSity of Miami NeURo-SPINAL INDEX

MOTOR EVALUATION FORM

MusCle Testing

Patient name:

Patient No.:

Date of admission:
Test performed by:

Date of test:

Level of injury:

Total

Head flexors

Head extensors

Left Right

Shoulder elevation

Arm elevation

Arm horizontal adduction

Elbow flexors

Wrist extensors

Elbow extensors

Wrist flexors

Extrinsic flexors

Extrinsic extensors

Thumb opposition

Finger abduction

Upper abdominals

Lower abdominals

Hip flexors

Hip extensors

Hip abductors

Knee flexors

Knee extensors

Foot dorsi-flexors

Foot everters

Foot flexors

Toe extensors

Grand total. 
monitored in an on-going fashion. Differential changes on any of the sub-scales can indicate the area affected by that intervention.

The UMNI was tested for inter-rater reliability utilising three physical therapists administering the test to Io patients at the University of Miami Spinal Cord Injury Centre, Rehabilitation facility.

The resulting multiple correlation coefficients were:

$$
\begin{array}{ll}
\text { Motor scale } & \text { Mult. } r=0.85 \\
\text { Sensory scale } & \text { Mult. } r=0.93
\end{array}
$$

The reliability tests were made on stabilised rehabilitation patients rather than acute patients to overcome the possibility of a change in neurological status during the time gap between assessments.

The high Inter-rater reliability can in part be attributed to the tests specific scoring instructions.

\section{Results}

Since April of 1978 over 50 patients have been tested in the emergency room and at designated follow-up time periods. This testing was part of a study to determine 'Outcome versus Emergency Care'. At the present time the populations for any of the critical time periods (i.e., 6 months, I year) are too small to make meaningful comparisons. However, it has been established that the initial tests can be performed within one hour of admission to the emergency room. Additionally, small changes have been noted in some patients over time.

\section{Discussion}

The UMNI provides a useful quantitative measure for the assessment of degree of spinal cord function. At present it can best be utilised as a research instrument to test the neurological effect of specific interventions following spinal cord injury. It can be used to document individual's changes or for statistical analyses comparing groups receiving different treatments. Adoption of this technique by other researchers will enable one to make meaningful comparisons of results. For statistical purposes the initial score is compared to subsequent scores to detect change. The use of change scores permits the grouping of patients displaying different initial degrees of severity. This method for score handling is used because the range of severity is large and the size of the patient population is relatively small.

One of the long range goals of this research group is to utilise the UMNI as a prognostic instrument. At present the prognosis for 'complete' injuries is usually poor, while 'incomplete' injuries are most often associated with some degree of neurological recovery. Once a sufficient data bank is established, it may be possible to discriminate between the types of injuries which have the highest probability of recovery as well as to actually estimate the degree of return of function.

It should be noted that the UMNI is not proposed as a substitute for, but rather as a complement to, other forms of testing. The UMNI assesses only spinal cord function and does not measure other important functions such as bowel and bladder control or psychological motivation. 


\section{REFERENCES}

BiCkerstafF, E. R. (1968). Neurological Examination in Clinical Practice. F. A. Davis Company, Philadelphia, $\mathrm{Pa}$.

BRACKen, M. B., WeBB, S. B., WAGNeR, F. C. (1977). Classification of the severity of acute spinal cord injury: implications for management. Paraplegia, 15, 3 I9.

Cheshire, D. J. E. (I970). A classification of the functional end-results of injury to the cervical spinal cord. Paraplegia, 8, 70.

GRANGER, C. V. (I973). A system for management of selected data in medical rehabilitation. A Monograph, Medical Rehabilitation Research and Training Center number 7, Tufts University School of Medicine, Boston, Massachusetts.

Jochreim, K. A. (1970). Problems of classification in traumatic paraplegia and tetraplegia. Paraplegia, 8, 80.

MAHONEY, F. \& BARTHEL, D. (1965). Functional evaluation: the Barthel index. Maryland State Medical fournal, 14 (2), 6I-65.

MiChAELIS, L. S. (I969). International inquiry on neurological terminology and prognosis in paraplegia and tetraplegia. Paraplegia, 7, I. 